membranes are stable over a wide range of $\mathrm{pH}$ values, and their size rejection curve is unusually steep compared with the rejection characteristics of polymer-based membranes.

Unfortunately, it was not possible to scale up the production of biologically derived membranes, so researchers and the membrane industry focused instead on the use of synthetic building blocks to make membranes with more uniform pores ${ }^{3}$. Some of the most successful of these synthetic strategies use self-assembly of block copolymer templates to generate a regular lamellar structure and then to convert it into a membrane by removing one of the polymer components. Takeji Hashimoto and colleagues at Kyoto University first demonstrated this process by synthesizing a microdomain-separated polystyrene-polyisoprene film and then degrading the polyisoprene component with ozonolysis to leave channels in the film ${ }^{4}$. Further refinements, such as the use of triblock copolymers, have increased the tunability and control over the system. Anne Mayes of the Massachusetts Institute of Technology and collaborators ${ }^{5}$ used a similar polymer-based approach to make a membrane that could cope with water fluxes (or permeances) as high as $91 \mathrm{~h}^{-1} \mathrm{~m}^{-2} \mathrm{bar}^{-1}$.

Ichinose and colleagues have combined these concepts in an experiment that uses self-assembly of globular proteins on sacrificial nanofilament templates to produce an extremely permeable and robust membrane (Fig. 1c) with rejection properties similar to ultrafiltration membranes with a low molecular mass cut-off. This fabrication technique is remarkably versatile and can use many different globular protein building blocks. The Japanese researchers chose ferritin because it produced membranes with very high permeances. They started by organizing ferritins on cadmium hydroxide nanostrands and then assembled them into a filter cake. After crosslinking the proteins they removed the metal hydroxide, leaving an extremely chemically resistant 60-nm-thick membrane that was stable in organic solvents and in a wide range of $\mathrm{pH}$ values (1.5 to 13 ). Remarkably, the pores were less than $2.2 \mathrm{~nm}$ across, and the water permeance could be as high as $9,0001 \mathrm{~h}^{-1} \mathrm{~m}^{-2}$ bar $^{-1}$.

Ichinose and colleagues also explored the rejection properties of their ferritin membranes using a wide range of analytes and solution conditions. Although more experiments will be needed to characterize the molecular mass cut-off completely, and to understand the rejection mechanisms in all their complexity, several trends are clear. First, it is evident that $\mathrm{pH}$ changes can modulate the pore size to some extent. Second, the shape of the molecule is important: TMPy, a square-planar porphyrin with a molecular mass of $\sim 680 \mathrm{Da}$, was almost completely rejected, whereas PSS, a linear polyelectrolyte with a molecular mass of $\sim 7,000 \mathrm{Da}$, passed through the membrane. It is possible that further engineering of ferritin or other protein building blocks could allow further fine-tuning of membrane cut-off and rejection properties.

Finally, the most remarkable and commercially attractive property of the ferritin-based membrane is its combination

of high permeance and low molecular mass cut-off (courtesy of the small pore size). Indeed, the membranes have permeances comparable to commercial ultrafiltration membranes that have much higher cut-offs, whereas commercial membranes with cutoffs comparable to the ferritin membranes have permeances that are more than two orders of magnitude lower. Ichinose and colleagues attribute this to the short (less than $6 \mathrm{~nm}$ in length) nanopores formed between the ferritin molecules.

This new class of protein membranes could potentially be a very attractive replacement for some conventional ultrafiltration and nanofiltration membranes. Better chemical stability may also open new opportunities for separation in the chemical industry. But it remains to be seen whether these protein membranes will be easier to manufacture than their S-layer membrane predecessors, as only a cost-effective manufacturing process will allow them to achieve their full potential in the marketplace.

Olgica Bakajin and Aleksandr Noy are at the Lawrence Livermore National Laboratory, 7000 East Avenue, Livermore, California 94550, USA. e-mail:bakajin1@llnl.gov;noy1@llnl.gov

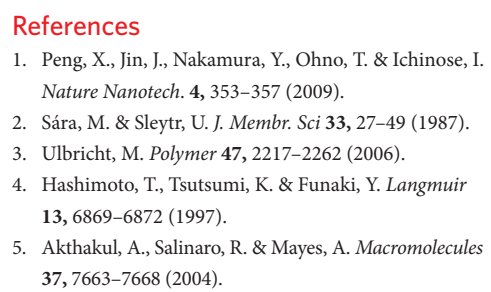

\title{
SUPERCONDUCTIVITY
}

\section{Flat out}

How thin can a film of lead be and still retain its ability to conduct electricity? Previous experiments have shown that films with just five layers of lead atoms become superconducting when they are cooled below about 6 K. Now Chih-Kang Shih and co-workers at the University of Texas at Austin have shown that just two layers of lead atoms - which contain just a single channel of quantum-well states can act as a superconductor (Science doi: 10.1126/science.1170775; 2009).

Shih and co-workers found that two layers of lead atoms on a silicon substrate can actually form two different types of lead film. As these scanning tunnelling micrographs show, type-I films have a $1 \times 1$ atomic structure and the same lattice parameter as bulk lead (left, scale

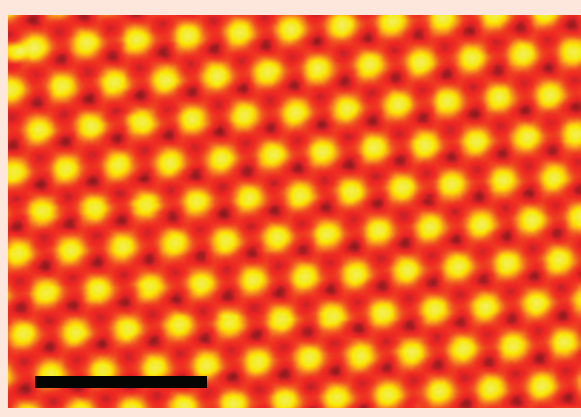

bar is $10 \mathrm{~nm}$ ), whereas type-II films have a $\sqrt{3} \times \sqrt{3}$ structure and a similar lattice constant to the silicon substrate (right). The films also have different transition temperatures $-4.9 \mathrm{~K}$ for type-I and $3.65 \mathrm{~K}$ for type-II - which the Texas team attribute to the type-II film experiencing tensile strain

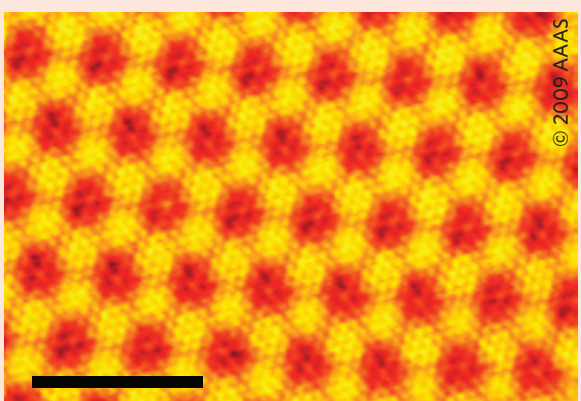

owing to its interaction with the substrate. Films containing three layers of lead atoms are not thermodynamically stable, whereas those containing four layers have a transition temperature of $6.7 \mathrm{~K}$.

PETER RODGERS 